PROCEEDINGS OF THE

AMERICAN MATHEMATICAL SOCIETY

Volume 138, Number 5, May 2010, Pages 1833-1841

S 0002-9939(09)10251-4

Article electronically published on December 31, 2009

\title{
THE PINCHING CONSTANT OF MINIMAL HYPERSURFACES IN THE UNIT SPHERES
}

\author{
QIN ZHANG
}

(Communicated by Richard A. Wentworth)

\begin{abstract}
In this paper, we prove that if $M^{n}(n \leq 8)$ is a closed minimal hypersurface in a unit sphere $S^{n+1}(1)$, then there exists a positive constant $\alpha(n)$ depending only on $n$ such that if $n \leq S \leq n+\alpha(n)$, then $M$ is isometric to a Clifford torus, where $S$ is the squared norm of the second fundamental form of $M$.
\end{abstract}

\section{INTRODUCTION}

Let $M^{n}$ be an $n$-dimensional closed minimal hypersurface in a unit sphere $S^{n+1}(1)$ of dimension $n+1$. Denote by $S$ the squared norm of the second fundamental form of $M^{n}$. Lawson [2, Simons [1] and Chern, do Carmo, Kobayashi [3] obtained independently the famous rigidity theorem, which says that if $S \leq n$, then $S \equiv 0$ or $S \equiv n$; i.e., $M^{n}$ is the great sphere $S^{n}(1)$ or the Clifford torus. Further discussions in this direction have been carried out by many other authors 6. 8, 9, 10, 11, 12, 13. In 4, Peng and Terng proved that if the scalar curvature of $M$ is constant, then there exists a positive constant $\alpha(n)$ depending only on $n$ such that if $n \leq S \leq n+\alpha(n)$, then $S \equiv n$. Later, Cheng and Yang [14] improved the pinching constant $\alpha(n)$ to $n / 3$. More generally, Peng and Terng [5] proved that if $M^{n}(n \leq 5)$ is a closed minimal hypersurface in $S^{n+1}$, then there exists a positive constant $\alpha(n)$ depending only on $n$ such that if $n \leq S \leq n+\alpha(n)$, then $S \equiv n$. So they proposed the following problem.

Let $M^{n}(n \geq 6)$ be a closed minimal hypersurface in $S^{n+1}$. Does there exist a positive constant $\alpha(n)$ depending only on $n$ such that if $n \leq S \leq n+\alpha(n)$, then $S \equiv n ?$

In [15, Cheng gives a positive answer under the additional condition that $M$ has only two distinct principal curvatures. Later, Cheng and Ishikawa 6] improved the result of Peng and Terng 5 when $n \leq 5$.

In this paper, we solve the problem proposed by Peng and Terng 5 for $n \leq 8$.

Theorem 1.1. Let $M^{n}(n \leq 8)$ be a closed minimal hypersurface in $S^{n+1}(1)$. If $n \leq S \leq n+\alpha(n)$, then $S \equiv n$ and $M^{n}$ is isometric to a Clifford torus $S^{m}\left(\sqrt{\frac{m}{n}}\right)$ $\times S^{n-m}\left(\sqrt{\frac{n-m}{n}}\right)$, where $\alpha(n)=\frac{2(n+4)(3-n \delta)}{9 n+30}, \delta(3)=0, \delta(4)=0.16, \delta(5)=$ $0.23, \delta(6)=0.28, \delta(7)=0.32$ and $\delta(8)=0.34$.

Received by the editors June 7, 2009, and, in revised form, August 18, 2009.

2000 Mathematics Subject Classification. Primary 53C40.

Key words and phrases. Minimal hypersurface, Clifford torus, second fundamental form. 
For $n \leq 5$, Cheng and Ishikawa [6] proved the following: Let $M$ be an $n$ dimensional ( $n \leq 5)$ closed minimal hypersurface of a unit sphere $S^{n+1}(1)$. If $n \leq$ $S \leq n+\alpha(n)$, then $S=n$, where $\alpha(3)=\frac{42}{85}, \alpha(4)=\frac{8}{31}$ and $\alpha(5)=\frac{3(21-5 \sqrt{17})}{28+3 \sqrt{17}}$. It is obvious that our pinching constant is larger than theirs. Up to now, the open problem for $n \geq 9$ is still open and it is a very hard problem.

\section{Fundamental FORMULAS}

Let $M^{n}$ be an $n$-dimensional hypersurface in an $(n+1)$-dimensional unit sphere $S^{n+1}(1)$. We choose a local orthonormal frame field $e_{1}, \cdots, e_{n+1}$ in $S^{n+1}(1)$, restricted to $M^{n}$, so that $e_{1}, \cdots, e_{n}$ are tangent to $M^{n}$. Let $\omega_{1}, \cdots, \omega_{n+1}$ denote the dual coframe field in $S^{n+1}(1)$. Then in $M^{n}, \omega_{n+1}=0$. It follows from Cartan's Lemma that

$$
\omega_{n+1 i}=\sum_{j} h_{i j} \omega_{j}
$$

The second fundamental form $\alpha$ and the mean curvature $H$ of $M^{n}$ are defined by

$$
\alpha=\sum_{i, j} h_{i j} \omega_{i} \omega_{j} e_{n+1}, \quad n H=\sum_{i} h_{i i},
$$

respectively. If $M^{n}$ is a minimal hypersurface, then $\sum_{i} h_{i i}=0$. The connection form $\omega_{i j}$ is characterized by the structure equations

$$
\begin{gathered}
d \omega_{i}+\sum_{j} \omega_{i j} \wedge \omega_{j}=0, \quad \omega_{i j}+\omega_{j i}=0, \\
d \omega_{i j}+\sum_{k} \omega_{i k} \wedge \omega_{k j}=\Omega_{i j}, \\
\Omega_{i j}=\frac{1}{2} \sum_{k, l} R_{i j k l} \omega_{k} \wedge \omega_{l},
\end{gathered}
$$

where $\Omega_{i j}$ (resp. $R_{i j k l}$ ) denotes the curvature form (resp. the components of the curvature tensor) of $M^{n}$. The Gauss equation is given by

$$
R_{i j k l}=\left(\delta_{i k} \delta_{j l}-\delta_{i l} \delta_{j k}\right)+\left(h_{i k} h_{j l}-h_{i l} h_{j k}\right) .
$$

Denote by $h_{i j k}, h_{i j k l}, h_{i j k l m}$ the components of the first, second and third covariant derivatives of the second fundamental form, respectively. Then

$$
\begin{gathered}
h_{i j k}=h_{i k j}=h_{j i k}, \\
h_{i j k l}-h_{i j l k}=\sum_{m} h_{i m} R_{m j k l}+\sum_{m} h_{m j} R_{m i k l}, \\
h_{i j k l m}-h_{i j k m l}=\sum_{r} h_{r j k} R_{r i l m}+\sum_{r} h_{i r k} R_{r j l m}+\sum_{r} h_{i j r} R_{r k l m} .
\end{gathered}
$$

For any fixed point $p$ in $M^{n}$, we take a local orthonormal frame field $e_{1}, \cdots, e_{n}$ such that

$$
h_{i j}= \begin{cases}\lambda_{i}, & i=j \\ 0, & i \neq j\end{cases}
$$


Let $S:=\sum_{i, j} h_{i j}^{2}=\sum_{i} \lambda_{i}^{2}$. The following formulas can be obtained by a direct computation (cf. [7]):

$$
\begin{gathered}
\frac{1}{2} \triangle S=\sum_{i, j, k} h_{i j k}^{2}-S(S-n), \\
\frac{1}{2} \sum_{i, j, k} h_{i j k}^{2}=\sum_{i, j, k, l} h_{i j k l}^{2}+(2 n+3-S) \sum_{i, j, k} h_{i j k}^{2}+3(2 B-A)-\frac{3}{2}|\nabla S|^{2},
\end{gathered}
$$

where $A=\sum_{i, j, k} \lambda_{i}^{2} h_{i j k}^{2}, B=\sum_{i, j, k} \lambda_{i} \lambda_{j} h_{i j k}^{2}$.

\section{Proof of Theorem 1.1}

At first we give a proposition and some lemmas which will play a crucial role in the proof of our theorem. For convenience, we let

$$
b_{i}=h_{i i 1}, b=\sum_{i \neq 1} b_{i}^{2}+\frac{1}{3} b_{1}^{2}, f=\sum_{i \neq 1}\left(\lambda_{1}^{2}-4 \lambda_{1} \lambda_{i}\right) b_{i}^{2}-\lambda_{1}^{2} b_{1}^{2} .
$$

Proposition 3.1. Let $M^{n}$ be a closed minimal hypersurface in $S^{n+1}(1)$. Suppose that

$$
3(A-2 B) \leq[2+\delta(n)] \sum_{i, j, k} h_{i j k}^{2},
$$

where $\delta(n)$ is a number depending only on $n$ such that $0 \leq \delta(n)<\min \left\{\frac{1}{2}, \frac{3}{n}\right\}$. Then there exists a positive constant $\alpha(n)$ depending only on $n$ such that if $n \leq$ $S \leq n+\alpha(n)$, then $S \equiv n$; i.e., $M^{n}$ is isometric to a Clifford torus $S^{m}\left(\sqrt{\frac{m}{n}}\right) \times$ $S^{n-m}\left(\sqrt{\frac{n-m}{n}}\right)$. Here, $\alpha(n)=\frac{2(n+4)(3-n \delta)}{9 n+30}$.

Proof. Since $M^{n}$ is a minimal hypersurface in $S^{n+1}(1)$, from (2.11) and (2.12) we have

$$
\begin{gathered}
\int_{M} \sum_{i, j, k} h_{i j k}^{2} d M=\int_{M} S(S-n) d M \\
-\frac{1}{2} \int_{M}|\nabla S|^{2} d M=\int_{M}\left[S \sum_{i, j, k} h_{i j k}^{2}-S^{2}(S-n)\right] d M \\
\left.\int_{M} \sum_{i, j, k, l} h_{i j k l}^{2} d M=\int_{M}\left[(S-2 n-3) \sum_{i, j, k} h_{i j k}^{2}+3(A-2 B)+\frac{3}{2}|\nabla S|^{2}\right)\right] d M .
\end{gathered}
$$

Letting $f_{3}=\sum_{i} \lambda_{i}^{3}$ and $f_{4}=\sum_{i} \lambda_{i}^{4}$, we have (cf. [6] $)$

$$
\begin{aligned}
& \sum_{i, j, k, l} h_{i j k l}^{2} \geq \frac{3}{2}\left(S f_{4}-f_{3}^{2}-2 S^{2}+n S\right)+\frac{3 S(S-n)^{2}}{2(n+4)}, \\
& \int_{M}(A-2 B) d M=\int_{M}\left[S f_{4}-f_{3}^{2}-S^{2}-\frac{1}{4}|\nabla S|^{2}\right] d M .
\end{aligned}
$$

From (3.3), (3.4) and (3.5), we have

$$
\int_{M}\left[(S-2 n-3) \sum_{i, j, k} h_{i j k}^{2}+\frac{3}{2}(A-2 B)+\frac{3}{2} S(S-n)+\frac{9}{8}|\nabla S|^{2}-\frac{3 S(S-n)^{2}}{2(n+4)}\right] d M \geq 0 .
$$


Noticing that $S^{2}=S(S-n)+n S$, from (3.1), (3.2) and (3.6), we have

$$
\int_{M}\left[\frac{3}{2}(A-2 B)+\frac{9 n+30}{4(n+4)} S(S-n)^{2}-\left(\frac{5}{4} S-\frac{n}{4}+\frac{3}{2}\right) \sum_{i, j, k} h_{i j k}^{2}\right] d M \geq 0 .
$$

Suppose $3(A-2 B) \leq[2+\delta(n)] S \sum_{i, j, k} h_{i j k}^{2}$ and $n \leq S \leq n+\alpha(n)$. From the above inequality, we have

$$
\int_{M}\left\{\frac{9 n+30}{4(n+4)} \alpha(n)-\frac{1-2 \delta(n)}{4}(S-n)-\frac{3-n \delta(n)}{2}\right\} \sum_{i, j, k} h_{i j k}^{2} d M \geq 0 .
$$

Since $\alpha(n)=\frac{2(n+4)(3-n \delta)}{9 n+30}$ and $\delta(n)<\min \left\{\frac{1}{2}, \frac{3}{n}\right\}$, we have

$$
-\int_{M}(S-n) \sum_{i, j, k} h_{i j k}^{2} d M \geq 0 .
$$

Hence, $S \equiv n$. This completes the proof of Proposition 3.1 .

Lemma 3.2. Let $M^{n}$ be a closed minimal hypersurface in $S^{n+1}(1)$. If $\lambda_{1}^{2}-$ $4 \lambda_{1} \lambda_{2} \geq t S(t \geq 2)$, then $\left(\lambda_{1}^{2}-4 \lambda_{1} \lambda_{2}\right)-\left(\lambda_{1}^{2}-4 \lambda_{1} \lambda_{i}\right) \geq r S(i \neq 1,2)$. Here, $r=\frac{16 t-8-12 \sqrt{-2 t^{2}+2 t+8}}{17}$.

Proof. Let $\lambda_{1}^{2}=x^{2} S, \lambda_{2}^{2}=y^{2} S(x, y>0)$. Since $\lambda_{1}^{2}-4 \lambda_{1} \lambda_{2} \geq t S(t \geq 2)$, we have $x^{2}+4 x y \geq t$, that is, $y \geq \frac{t-x^{2}}{4 x}$. Hence, we have

$$
\begin{aligned}
\lambda_{1}^{2}-4 \lambda_{1} \lambda_{i} & \leq\left(x^{2}+4 x \sqrt{1-x^{2}-y^{2}}\right) S \\
& \leq\left\{x^{2}+\sqrt{16 x^{2}-16 x^{4}-\left(t-x^{2}\right)^{2}}\right\} S \\
& =\left\{x^{2}+\sqrt{-17 x^{4}+(16+2 t) x^{2}-t^{2}}\right\} S .
\end{aligned}
$$

Let $g(z)=z+\sqrt{-17 z^{2}+(16+2 t) z-t^{2}}(0<z<1)$. Then

$$
g^{\prime}(z)=1-\frac{17 z-(8+t)}{\sqrt{-17 z^{2}+(16+2 t) z-t^{2}}} .
$$

Letting $g^{\prime}\left(z_{0}\right)=0$, we have

$$
z_{0}=\frac{3(8+t)+2 \sqrt{-2 t^{2}+2 t+8}}{51} .
$$

Hence we have

$$
g(z) \leq g\left(z_{0}\right)=\frac{t+8+12 \sqrt{-2 t^{2}+2 t+8}}{17},
$$

which implies that

$$
\lambda_{1}^{2}-4 \lambda_{1} \lambda_{i} \leq \frac{t+8+12 \sqrt{-2 t^{2}+2 t+8}}{17} .
$$

Since $\lambda_{1}^{2}-4 \lambda_{1} \lambda_{2} \geq t S$, we have

$$
\left(\lambda_{1}^{2}-4 \lambda_{1} \lambda_{2}\right)-\left(\lambda_{1}^{2}-4 \lambda_{1} \lambda_{i}\right) \geq \frac{16 t-8-12 \sqrt{-2 t^{2}+2 t+8}}{17} .
$$

This completes the proof of Lemma 3.2 
Lemma 3.3. Let $f_{n}(t)=17[t-2-\delta(n)][3(n-2) t+(n+2) \delta(n)+10-4 n]$ and $g_{n}(t)=[8+16 \delta(n)]\left(4 t-2-3 \sqrt{-2 t^{2}+2 t+8}\right)$. Then

$$
h_{n}(t)=f_{n}(t)-g_{n}(t) \leq 0 \quad(t \geq 2,4 \leq n \leq 8) .
$$

Here, $\delta(4)=0.16, \delta(5)=0.23, \delta(6)=0.28, \delta(7)=0.32$ and $\delta(8)=0.34$.

Proof. By a direct computation, we obtain

$$
h_{n}^{\prime \prime}(t)=51\left\{2(n-2)-[8+16 \delta(n)]\left(-2 t^{2}+2 t+8\right)^{-\frac{3}{2}}\right\}
$$

and

$$
h_{n}^{\prime \prime \prime}(t)=-153[8+16 \delta(n)](2 t-1)\left(-2 t^{2}+2 t+8\right)^{-\frac{5}{2}}<0 \quad(t \geq 2) .
$$

On the other hand, $h_{n}^{\prime \prime}(2)>0$ and $h_{n}(2)<0$. Hence, if there exist real numbers $t_{i}>2(i=1,2)$ such that $h_{n}^{\prime}\left(t_{1}\right)>0, h_{n}^{\prime}\left(t_{2}\right)<0$ and $h_{n}(t) \leq 0\left(t_{1} \leq t \leq t_{2}\right)$, then $h_{n}(t) \leq 0(\forall t \geq 2)$.

In the case $n=4$, since $\delta(4)=0.16$, we have

$$
\begin{array}{r}
f_{4}(t)=17\left(6 t^{2}-18 t+10.8864\right), \\
g_{4}(t)=10.56\left(4 t-2-3 \sqrt{-2 t^{2}+2 t+8}\right), \\
h_{4}^{\prime}(t)=204 t-348.24-31.68(2 t-1)\left(-2 t^{2}+2 t+8\right)^{-\frac{1}{2}} .
\end{array}
$$

By a direct computation, we obtain

$$
h_{4}^{\prime}(2.48)>0, h_{4}^{\prime}(2.5)<0 .
$$

On the other hand, when $2.48 \leq t \leq 2.5$, we have

$$
f_{4}(t) \leq f_{4}(2.5) \leq 57.6, g_{4}(t) \geq g_{4}(2.48) \geq 57.8 .
$$

This implies that

$$
h_{4}(t) \leq 0(2.48 \leq t \leq 2.5) .
$$

From (3.10) and (3.11), we know that Lemma 3.3 is true in the case $n=4$.

In the case $n=5$, since $\delta(5)=0.23$, we have

$$
\begin{array}{r}
f_{5}(t)=17\left(9 t^{2}-28.46 t+18.7097\right), \\
g_{5}(t)=11.68\left(4 t-2-3 \sqrt{-2 t^{2}+2 t+8}\right), \\
h_{5}^{\prime}(t)=306 t-530.54-35.04(2 t-1)\left(-2 t^{2}+2 t+8\right)^{-\frac{1}{2}} .
\end{array}
$$

By a direct computation, we obtain

$$
h_{5}^{\prime}(2.51)>0, h_{5}^{\prime}(2.52)<0 .
$$

On the other hand, when $2.51 \leq t \leq 2.52$, we have

$$
f_{5}(t) \leq f_{5}(2.52) \leq 70.5, g_{5}(t) \geq g_{5}(2.51) \geq 71 .
$$

This implies that

$$
h_{5}(t) \leq 0(2.51 \leq t \leq 2.52) .
$$

From (3.12) and (3.13), we know that Lemma 3.3 is true in the case $n=5$.

In the case $n=6$, since $\delta(6)=0.28$, we have

$$
\begin{array}{r}
f_{6}(t)=17\left(12 t^{2}-39.12 t+26.8128\right), \\
g_{6}(t)=12.48\left(4 t-2-3 \sqrt{-2 t^{2}+2 t+8}\right), \\
h_{6}^{\prime}(t)=408 t-714.96-37.44(2 t-1)\left(-2 t^{2}+2 t+8\right)^{-\frac{1}{2}} .
\end{array}
$$


By a direct computation, we obtain

$$
h_{6}^{\prime}(2.53)>0, h_{6}^{\prime}(2.535)<0 .
$$

On the other hand, when $2.53 \leq t \leq 2.535$, we have

$$
f_{6}(t) \leq f_{6}(2.535) \leq 81, g_{6}(t) \geq g_{6}(2.51) \geq 82 .
$$

This implies that

$$
h_{6}(t) \leq 0(2.53 \leq t \leq 2.535) .
$$

From (3.14) and (3.15), we know that Lemma 3.3 is true in the case $n=6$.

In the case $n=7$, since $\delta(7)=0.32$, we have

$$
\begin{array}{r}
f_{7}(t)=17\left(15 t^{2}-49.92 t+35.0784\right), \\
g_{7}(t)=13.12\left(4 t-2-3 \sqrt{-2 t^{2}+2 t+8}\right), \\
h_{7}^{\prime}(t)=510 t-901.12-39.36(2 t-1)\left(-2 t^{2}+2 t+8\right)^{-\frac{1}{2}} .
\end{array}
$$

By a direct computation, we obtain

$$
h_{7}^{\prime}(2.54)>0, h_{7}^{\prime}(2.544)<0 .
$$

On the other hand, when $2.54 \leq t \leq 2.544$, we have

$$
f_{7}(t) \leq f_{7}(2.544) \leq 88, g_{7}(t) \geq g_{7}(2.54) \geq 90 .
$$

This implies that

$$
h_{7}(t) \leq 0(2.54 \leq t \leq 2.544) .
$$

From (3.16) and (3.17), we know that Lemma 3.3 is true in the case $n=7$.

In the case $n=8$, since $\delta(8)=0.34$, we have

$$
\begin{array}{r}
f_{8}(t)=17\left(18 t^{2}-60.72 t+43.524\right), \\
g_{8}(t)=13.44\left(4 t-2-3 \sqrt{-2 t^{2}+2 t+8}\right), \\
h_{8}^{\prime}(t)=612 t-1086-40.32(2 t-1)\left(-2 t^{2}+2 t+8\right)^{-\frac{1}{2}} .
\end{array}
$$

By a direct computation, we obtain

$$
h_{8}^{\prime}(2.5465)>0, h_{8}^{\prime}(2.5468)<0 .
$$

On the other hand, when $2.5465 \leq t \leq 2.5468$, we have

$$
f_{8}(t) \leq f_{8}(2.5468) \leq 95.78, g_{8}(t) \geq g_{8}(2.5465) \geq 95.8 .
$$

This implies that

$$
h_{8}(t) \leq 0(2.5465 \leq t \leq 2.5468) .
$$

From (3.18) and (3.19), we know that Lemma 3.3 is true in the case $n=8$. This completes the proof of Lemma 3.3

Lemma 3.4. Let $M^{n}$ be a closed minimal hypersurface in $S^{n+1}(1)$. Then

$$
f \leq[2+\delta(n)] S b, 3 \leq n \leq 8 .
$$

Here, $\delta(3)=0, \delta(4)=0.16, \delta(5)=0.23, \delta(6)=0.28, \delta(7)=0.32$ and $\delta(8)=0.34$. 
Proof. In the case $n=3$, if $b_{1}=0$, then $b_{2}^{2}=b_{3}^{2}=\frac{1}{2} b$. Hence

$$
\begin{aligned}
f & =\frac{1}{2}\left(\lambda_{1}^{2}-4 \lambda_{1} \lambda_{2}+\lambda_{1}^{2}-4 \lambda_{1} \lambda_{3}\right) b \\
& =\left\{\lambda_{1}^{2}-2 \cdot \frac{\lambda_{1}}{\sqrt{2}} \cdot \sqrt{2} \lambda_{2}-2 \cdot \frac{\lambda_{1}}{\sqrt{2}} \cdot \sqrt{2} \lambda_{3}\right\} b \\
& \leq 2\left(\lambda_{1}^{2}+\lambda_{2}^{2}+\lambda_{3}^{2}\right) b \leq 2 S b .
\end{aligned}
$$

When $b_{1} \neq 0$, we let $b_{2}=\left(x-\frac{1}{2}\right) b_{1}$ and $\lambda_{2}=\left(y-\frac{1}{2}\right) \lambda_{1}$. Then

$$
\begin{aligned}
f & =\frac{36 x^{2}+48 x y+3}{48 x^{2} y^{2}+36 x^{2}+20 y^{2}+15} \cdot 2 S b \\
& =\frac{36 x^{2}+48 x y+3}{36 x^{2}+48 x y+3+48(x y-1 / 2)^{2}+20 y^{2}} \cdot 2 S b \\
& \leq 2 S b .
\end{aligned}
$$

From the above discussion, we know that Lemma 3.4 is true in the case $n=3$.

In the case $4 \leq n \leq 8$, if $\lambda_{1}^{2}-4 \lambda_{1} \lambda_{i} \leq[2+\delta(n)] S(\forall i \neq 1)$, then Lemma 3.4 is true. Otherwise, without loss of generality, we suppose that $\lambda_{1}^{2}-4 \lambda_{1} \lambda_{2}=t S(t \geq 2)$ and $b_{1}=x b_{2}$. Then

$$
\sum_{i \neq 1,2} b_{i}^{2} \geq \frac{(1+x)^{2}}{n-2} b_{2}^{2}, \lambda_{1}^{2} \geq(t-2) S .
$$

From the above inequalities and Lemma 3.2, we have

$$
\begin{aligned}
f-[2+\delta(n)] S b \leq & {[t-2-\delta(n)] S b_{2}^{2}+[t-r-2-\delta(n)] S \sum_{i \neq 1,2} b_{i}^{2} } \\
& -\left\{t-2+\frac{2+\delta(n)}{3}\right\} S b_{1}^{2} \\
\leq & {[t-2-\delta(n)] S b_{2}^{2}+\frac{t-r-2-\delta(n)}{n-2}(1+x)^{2} S b_{2}^{2} } \\
& -\left\{t-2+\frac{2+\delta(n)}{3}\right\} x^{2} S b_{2}^{2} .
\end{aligned}
$$

Here, $r=\frac{16 t-8-12 \sqrt{-2 t^{2}+2 t+8}}{17}$.

Let $F(n, t, x)=t-2-\delta(n)+\frac{t-r-2-\delta(n)}{n-2}(1+x)^{2}-\left\{t-2+\frac{2+\delta(n)}{3}\right\} x^{2}$.

The above inequality becomes

$$
f-[2+\delta(n)] S b \leq F(n, t, x) S b_{2}^{2}
$$

Since

$$
\frac{1}{2} \frac{\partial F(n, t, x)}{\partial x}=\frac{t-r-2-\delta(n)}{n-2}(1+x)-\left\{t-2+\frac{2+\delta(n)}{3}\right\} x
$$

we have

$$
\begin{aligned}
F(n, t, x) & \leq F\left(n, t, x_{0}\right) \\
& =\frac{t-2-\delta(n)}{G}\left\{(n-2) t+\frac{(n+2) \delta(n)+10-4 n}{3}\right\}-\frac{2+4 \delta(n)}{3 G} r .
\end{aligned}
$$


Here, $-x_{0}=\frac{3[r+2+\delta(n)-t]}{3 r+3(n-3) t+14+(n+1) \delta(n)-4 n}, \frac{\partial F\left(n, t, x_{0}\right)}{\partial x}=0$, and $G=$ $r+2+\delta(n)-t+(n-2)\left(t-2+\frac{2+\delta(n)}{3}\right)$.

Notice that $h_{n}(t)=51 F\left(n, t, x_{0}\right) G$, where $h_{n}(t)$ is defined as in Lemma 3.3. From (3.20), (3.21) and Lemma 3.3, we have

$$
f \leq[2+\delta(n)] S b .
$$

This completes the proof of Lemma 3.4

Now we are in a position to give the proof of Theorem 1.1

Proof of Theorem 1.1. From Lemma 3.4 we have obtained

$$
f=\sum_{i \neq 1}\left(\lambda_{1}^{2}-4 \lambda_{1} \lambda_{i}\right) h_{i i 1}^{2}-\lambda_{1}^{2} h_{111}^{2} \leq(2+\delta) S\left(\sum_{i \neq 1} h_{i i 1}^{2}+\frac{1}{3} h_{111}^{2}\right) .
$$

In general,

$$
f_{j}=\sum_{i \neq j}\left(\lambda_{j}^{2}-4 \lambda_{i} \lambda_{j}\right) h_{i i j}^{2}-\lambda_{j}^{2} h_{j j j}^{2} \leq(2+\delta) S\left(\sum_{i \neq j} h_{i i j}^{2}+\frac{1}{3} h_{j j j}^{2}\right), \forall j .
$$

Hence we get

$$
\begin{aligned}
3(A-2 B)= & \sum_{i \neq j \neq k \neq i}\left[2\left(\lambda_{i}^{2}+\lambda_{j}^{2}+\lambda_{k}^{2}\right)-\left(\lambda_{i}+\lambda_{j}+\lambda_{k}\right)^{2}\right] h_{i j k}^{2} \\
& -3 \sum_{i} \lambda_{i}^{2} h_{i i i}^{2}+3 \sum_{i \neq j}\left(\lambda_{j}^{2}-4 \lambda_{i} \lambda_{j}\right) h_{i i j}^{2} \\
\leq & 2 S \sum_{i \neq j \neq k \neq i} h_{i j k}^{2}+3 \sum_{i \neq j}\left[\left(\lambda_{j}^{2}-4 \lambda_{i} \lambda_{j}\right) h_{i i j}^{2}-\lambda_{j}^{2} h_{j j j}^{2}\right] \\
\leq & (2+\delta) S\left\{\sum_{i \neq j \neq k \neq i} h_{i j k}^{2}+3 \sum_{i \neq j} h_{i i j}^{2}+\sum_{j} h_{j j j}^{2}\right\} \\
= & (2+\delta) S \sum_{i, j, k} h_{i j k}^{2} .
\end{aligned}
$$

Notice that $\delta(3)=0, \delta(4)=0.16, \delta(5)=0.23, \delta(6)=0.28, \delta(7)=0.32, \delta(8)=0.34$ and $\alpha(n)=\frac{2(n+4)(3-n \delta)}{9 n+30}$. We conclude from Proposition 3.1 that $S \equiv n$. This completes the proof of Theorem 1.1

\section{REFERENCES}

1. J. Simons, Minimal varieties in Riemannian manifolds, Ann. of Math. (2), 88 (1968), 62-105. MR0233295 (38:1617)

2. H. B. Lawson Jr., Local rigidity theorems for minimal hypersurfaces, Ann. of Math. (2), 89 (1969), 187-197. MR0238229 (38:6505)

3. S. S. Chern, M. do Carmo, S. Kobayashi, Minimal submanifolds of a sphere with second fundamental form of constant length, Functional analysis and related fields, Springer, New York, 1970, pp. 59-75. MR0273546 (42:8424)

4. C. K. Peng, C. L. Terng, Minimal hypersurfaces of spheres with constant scalar curvature, Ann. of Math. Stud., 103, Princeton University Press, 1983, 179-198. MR795235 (87k:53143)

5. C. K. Peng, C. L. Terng, The scalar curvature of minimal hypersurfaces in spheres, Math. Ann., 266 (1983), 105-113. MR722930 (85c:53099)

6. Q. M. Cheng, S. Ishikawa, A characterization of the Clifford torus, Proc. Amer. Math. Soc., 127 (1999), 819-828. MR1636934 (99g:53064) 
7. Q. M. Cheng, The classification of complete hypersurfaces with nonzero constant mean curvature of space form of dimension 4, Mem. Fac. Sci. Kyushu Univ. Ser. A, 47 (1993), 79-102. MR:1222356 (94h:53067)

8. S. Y. Cheng, S. T. Yau, Hypersurfaces with constant scalar curvature, Math. Ann., 225 (1977), no. 3, 195-204. MR0431043 (55:4045)

9. A. M. Li, J. M. Li, An intrinsic rigidity theorem for minimal submanifolds in a sphere, Arch. Math. (Basel), 58 (1992), no. 6, 582-594. MR.1161925 (93b:53050)

10. H. W. Xu, A rigidity theorem for submanifolds with parallel mean curvature in a sphere, Arch. Math. (Basel), 61 (1993), no. 5, 489-496. MR1241055 (94m:53084)

11. H. W. Xu, On closed minimal submanifolds in pinched Riemannian manifolds, Trans. Amer. Math. Soc., 347 (1995), no. 5, 1743-1751. MR1243175 (95h:53088)

12. H. W. Xu, W. Fang, F. Xiang, A generalization of Gauchman's rigidity theorem, Pacific J. Math., 228 (2006), no. 1, 185-199. MR2263029 (2007j:53048)

13. S. T. Yau, Submanifolds with constant mean curvature. I, II, Amer. J. Math., 96 (1974), 346-366; ibid., 97 (1975), 76-100. MR0370443 (51:6670)

14. Q. M. Cheng, H. C. Yang, Chern's conjecture on minimal hypersurfaces, Math. Z., 227 (1998), no. 3, 377-390. MR.1612653 (99c:53070)

15. Q. M. Cheng, The rigidity of Clifford torus $S^{1}\left(\sqrt{\frac{1}{n}}\right) \times S^{n-1}\left(\sqrt{\frac{n-1}{n}}\right)$, Comment. Math. Helvetici, 71 (1996), 60-69. MR 1371678 (97a:53094)

16. Y. T. Zhang, S. L. Xu, Rigidity of the Clifford torus $S^{m}(\sqrt{m / n}) \times S^{n-m}(\sqrt{(n-m) / n})$, Acta Mathematica Scientia Ser. A Chin. Ed., 28 (2008), 128-132. MR2392041 (2008m:53145)

Institute of Mathematics and Software Science, Sichuan Normal University, Chengdu 610066, People's Republic of China

E-mail address: zhangdiligence@126.com 